\title{
A Parallel Distance-2 Graph Coloring Algorithm for Distributed Memory Computers ${ }^{\star}$
}

\author{
Doruk Bozdağ $^{1}$, Umit Catalyurek ${ }^{1}$, Assefaw H. Gebremedhin ${ }^{2}$, Fredrik Manne ${ }^{3}$, \\ Erik G. Boman ${ }^{4}$, and Füsun Özgüner ${ }^{1}$ \\ 1 Ohio State University, USA \\ \{bozdagd, ozguner\}@ece.osu.edu, umit@bmi.osu.edu \\ 2 Old Dominion University, USA \\ assefaw@Cs . odu . edu \\ 3 University of Bergen, Norway \\ Fredrik. Mannedii.uib.no \\ 4 Sandia National Laboratories, USA \\ egboman@sandia.gov
}

\begin{abstract}
The distance-2 graph coloring problem aims at partitioning the vertex set of a graph into the fewest sets consisting of vertices pairwise at distance greater than two from each other. Application examples include numerical optimization and channel assignment. We present the first distributed-memory heuristic algorithm for this NP-hard problem. Parallel speedup is achieved through graph partitioning, speculative (iterative) coloring, and a BSP-like organization of computation. Experimental results show that the algorithm is scalable, and compares favorably with an alternative approach—solving the problem on a graph $G$ by first constructing the square graph $G^{2}$ and then applying a parallel distance-1 coloring algorithm on $G^{2}$.
\end{abstract}

\section{Introduction}

An archetypal problem in the efficient computation of sparse Jacobian and Hessian matrices is the distance-2 (D2) vertex coloring problem in an appropriate graph [1]. D2 coloring also finds applications in channel assignment [2] and facility location problems [3]. It is closely related to a strong coloring of a hypergraph which in turn models problems that arise in the design of multifiber WDM networks [4]. The D2 coloring problem is known to be NP-hard [5].

In many parallel applications where a graph coloring is required, the graph is already distributed among processors. Under such circumstances, gathering the graph on one processor to perform the coloring may not be feasible due to memory constraints. Moreover, in some parallel applications the coloring needs to be performed repeatedly

\footnotetext{
* This work was supported in part by NSF grants ACI-0203722, ACI-0203846, ANI-0330612, CCF-0342615, CNS-0426241, NIH NIBIB BISTI P20EB000591, Ohio Board of Regents BRTTC BRTT02-0003, Ohio Supercomputing Center PAS0052, and SNL Doc.No: 283793. Sandia is a multiprogram laboratory operated by Sandia Corporation, a Lockheed Martin company, for the U.S. DOE's National Nuclear Security Administration under contract DE-AC0494AL85000.
} 
due to changes in the structure of the graph. Here, the coloring may take up a substantial amount of overall computation time unless a scalable algorithm is used.

A number of papers dealing with the design of efficient parallel distance-1 (D1) coloring algorithms have appeared [67/89]. For D2 coloring we are not aware of any work other than [10] where an algorithm for shared memory computers was presented.

In this paper, we present an efficient parallel D2 coloring algorithm suitable for distributed memory computers. The algorithm is an extension of the parallel D1 coloring algorithm presented in [6]. The latter is an iterative data parallel algorithm that proceeds in two-phased rounds. In the first phase, processors concurrently color the vertices assigned to them. Adjacent vertices colored in the same parallel step of this phase may result in inconsistencies. In the second phase, processors concurrently check the validity of the colors assigned to their respective vertices and identify a set of vertices that needs to be re-colored in the next round to resolve the detected inconsistencies. The algorithm terminates when every vertex has been colored correctly. To reduce communication frequency, the coloring phase is further decomposed into computation and communication sub-phases. During a computation sub-phase, a group of vertices, rather than a single vertex, is colored based on currently available color information. In a communication sub-phase processors exchange recent color information.

The key issue in extending this approach to the D2 coloring case is devising an efficient means of information exchange between processors hosting a pair of vertices that are two edges away from each other. We use a scheme in which the host processor of a vertex $v$ is responsible for (i) coloring $v$, (ii) relaying color information to processors that store the D1 neighbors of $v$, and (iii) detecting inconsistencies that involve the D1 neighbors of $v$.

Our parallel D2 coloring algorithm has been implemented using MPI. Results from experiments performed on a 32-node PC cluster using a number of real-world as well as random graphs show that the algorithm is efficient and scalable. We have also compared our D2 coloring algorithm on a given graph $G$ with the parallel D1 coloring algorithm from [6] applied to the square graph $G^{2}$. These results in general show that our algorithm scales better and uses less memory and storage.

In the sequel, we discuss preliminary concepts in Section 2, present our algorithm in Section 3, report experimental results in Section 4 and conclude in Section 5.

\section{Distance-2 Graph and Hypergraph Coloring}

Two distinct vertices in a graph $\mathcal{G}=(\mathcal{V}, \mathcal{E})$ are distance- $k$ neighbors if the shortest path connecting them consists of at most $k$ edges. A distance- $k$ coloring of $\mathcal{G}=(\mathcal{V}, \mathcal{E})$ is a mapping $C: \mathcal{V} \rightarrow\{1,2, \ldots, q\}$ such that $C(v) \neq C(w)$ whenever vertices $v$ and $w$ are distance- $k$ neighbors. The associated optimization problem aims at minimizing $q$. A distance- $k$ coloring of a graph $\mathcal{G}=(\mathcal{V}, \mathcal{E})$ is equivalent to a D1 coloring of the $k$ th power graph $\mathcal{G}^{k}=(\mathcal{V}, \mathcal{F})$ where $(v, w) \in \mathcal{F}$ whenever vertices $v$ and $w$ are distance- $k$ neighbors in $\mathcal{G}$. We denote the set of distance- $k$ neighbors of vertex $v$ by $N_{k}(v)$, and the set $N_{k}(v) \cup\{v\}$ by $N_{k}[v]$. For simplicity, we drop the subscript in the case where $k=1$.

Let $A$ be a symmetric matrix with nonzero diagonal elements and $\mathcal{G}_{a}(A)=(\mathcal{V}, \mathcal{E})$ be the adjacency graph of $A$, where $\mathcal{V}$ corresponds to the columns of $A$. As illustrated 
$\left[\begin{array}{ccccc}\mathrm{a}_{11} & 0 & 0 & 0 & \mathrm{a}_{15} \\ 0 & \mathrm{a}_{22} & \mathrm{a}_{23} & 0 & 0 \\ 0 & \mathrm{a}_{32} & \mathrm{a}_{33} & \mathrm{a}_{34} & \mathrm{a}_{35} \\ 0 & 0 & \mathrm{a}_{43} & \mathrm{a}_{44} & \mathrm{a}_{45} \\ \mathrm{a}_{51} & 0 & \mathrm{a}_{53} & \mathrm{a}_{54} & \mathrm{a}_{55}\end{array}\right]$

A

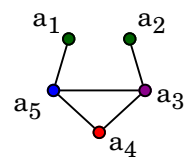

$G_{a}$

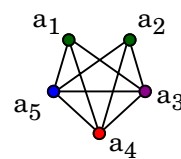

$G_{a}^{2}$ $\left[\begin{array}{ccccc}\mathrm{a}_{11} & \mathrm{a}_{12} & 0 & 0 & \mathrm{a}_{15} \\ \mathrm{a}_{21} & \mathrm{a}_{22} & 0 & 0 & 0 \\ \mathrm{a}_{31} & 0 & 0 & \mathrm{a}_{34} & 0 \\ 0 & 0 & \mathrm{a}_{43} & \mathrm{a}_{44} & \mathrm{a}_{45}\end{array}\right]$

A

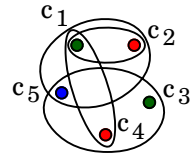

$H$

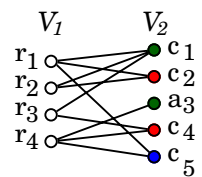

$G_{b}$

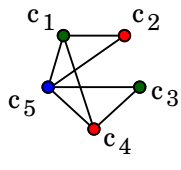

$G_{b}^{2}\left[V_{2}\right]$

Fig. 1. Equivalence among structurally orthogonal column partition of $A$, D2 coloring of $\mathcal{G}(A)$ and D1 coloring of $\mathcal{G}^{2}(A)$. Top: symmetric case. Bottom: non-symmetric case (also shows equivalence with strong coloring of hypergraph $H$ ).

in the upper row of Figure 1, a partitioning of the columns of $A$ into groups of structurally orthogonal columns is equivalent to a $\mathrm{D} 2$ coloring of $\mathcal{G}_{a}(A)$. (Two columns are structurally orthogonal if they do not have nonzero entries in the same row.) The right most subfigure in Figure 1 shows the equivalent D1 coloring in the square graph $\mathcal{G}_{a}^{2}$.

Now let $A$ be non-symmetric. The bipartite graph of $A$ is the graph $\mathcal{G}_{b}=\left(\mathcal{V}_{1}, \mathcal{V}_{2}, \mathcal{E}\right)$ where $\mathcal{V}_{1}$ is the row vertex set, $\mathcal{V}_{2}$ is the column vertex set, and there exits an edge between row vertex $r_{i}$ and column vertex $c_{j}$ whenever $a_{i j} \neq 0$. As the lower row of Figure 1 1llustrates, a partitioning of the columns of $A$ into groups of structurally orthogonal columns is equivalent to a partial D2 coloring of $\mathcal{G}_{b}(A)$ on $\mathcal{V}_{2}$. The right most subfigure shows the equivalent D1 coloring of $\mathcal{G}_{b}^{2}\left[\mathcal{V}_{2}\right]$, the subgraph of the square graph $\mathcal{G}_{b}^{2}$ induced by $\mathcal{V}_{2}$.

D2 coloring of a bipartite graph is also related to a variant of hypergraph coloring. A hypergraph $\mathcal{H}=(\mathcal{V}, \mathcal{E})$ consists of a vertex set $\mathcal{V}$ and a collection $\mathcal{E}$ of subsets of $\mathcal{V}$ called hyperedges. A strong hypergraph coloring is a mapping $C: \mathcal{V} \rightarrow\{1,2, \ldots, q\}$ such that $C(v) \neq C(v)$ whenever $\{v, w\} \subseteq e \in \mathcal{E}$. As Figure 1 illustrates, a strong coloring of a hypergraph is equivalent to a partial D2 coloring of its hyperedge-vertex incidence bipartite graph. For further discussion on the equivalence among matrix partitioning, D2 graph coloring and hypergraph coloring as well as their relationships to computation of Jacobians and Hessians, see [1].

\section{Parallel Distance-2 Coloring}

In this section we describe our new parallel D2 coloring algorithm for a general graph $\mathcal{G}=(\mathcal{V}, \mathcal{E})$. Initially, the input graph is assumed to be distributed among $p$ processors. The set $V_{i}$ of vertices in the partition $\left\{V_{1}, \ldots, V_{p}\right\}$ of $\mathcal{V}$ is assigned to and colored by processor $P_{i}$; we say that $P_{i}$ owns $V_{i} . P_{i}$ also stores the adjacency list of its vertices and the IDs of the processors owning them. This classifies $\mathcal{V}$ into interior and boundary 


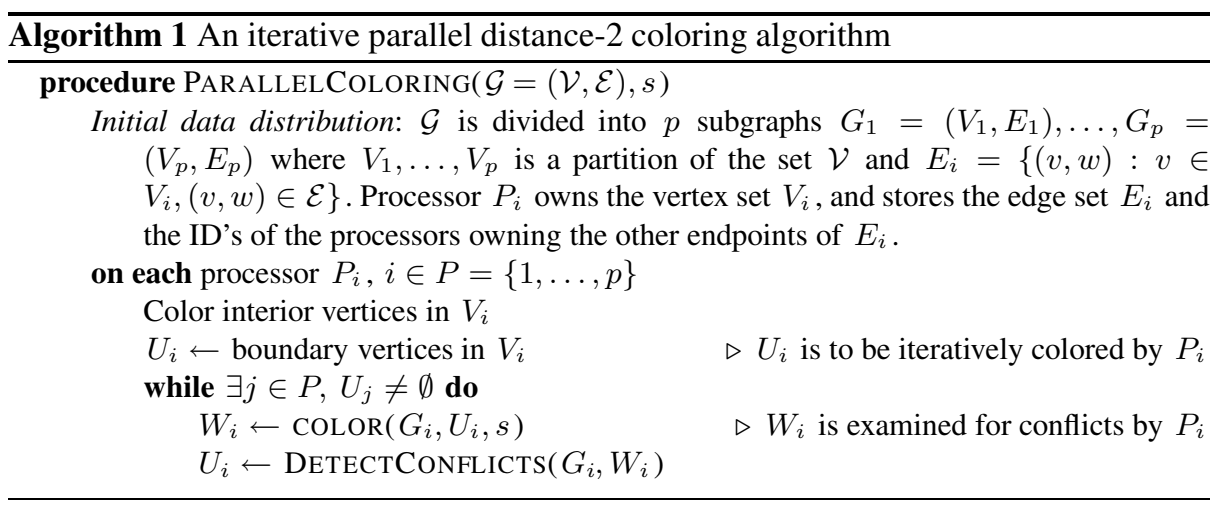

vertices. All D1 neighbors of an interior vertex are owned by the same processor as itself. A boundary vertex has at least one D1 neighbor owned by a different processor.

Clearly, any pair of interior vertices, that are assigned to different processors, can safely be colored concurrently. This is not true for a pair containing a boundary vertex. In particular, if such a pair is colored at the same parallel superstep, then the partners may receive the same color and result in a conflict. However, if we enforce that interior vertices be colored before or after boundary vertices, then a conflict can only occur for pairs of boundary vertices. Thus, the presented algorithm is concerned with parallel coloring of boundary vertices.

The main idea in our algorithm is to color boundary vertices concurrently in a speculative manner and then detect and rectify conflicts that may have arisen. The algorithm is iterative-it proceeds in rounds. Each round consists of a tentative coloring and a conflict detection phase. Both of these phases are performed in parallel. The latter phase detects conflicts in a current coloring and accumulates a list of vertices to be recolored in the next round. Given a pair of vertices involved in a conflict, only one of them needs to be recolored to resolve the conflict; the choice is done randomly. The algorithm terminates when there are no more vertices to be colored. The high-level structure of the algorithm is outlined in Algorithm 1 .

Notice the termination condition of the while-loop. Even if a processor $P_{i}$ currently has no vertices to color $\left(U_{i}=\emptyset\right)$, it could still be active since other processors may require color information from $P_{i}$. Furthermore, $P_{i}$ may participate in detecting conflicts on other processors.

For every path $v, w, x$, the host processor for vertex $w$ is responsible for detecting D2 conflicts that involve vertices $v$ and $x$ as well as D1 conflicts involving $w$ and its adjacent vertices $N(w)$. The set $W_{i}$ in Algorithm 1 contains the current set of vertices residing on processor $P_{i}$ that will be examined for detecting these conflicts. $W_{i}$ includes both 'middle' vertices, and vertices from $U_{i}$. The discussion of the routines COLOR and DETECTCONFLICTS in Sections 3.1 and 3.2 will clarify these points.

\subsection{The Tentative Coloring Phase}

The tentative coloring phase is organized as a sequence of supersteps. In each superstep, each processor colors $s$ vertices sequentially, and sends the colors of these vertices to 


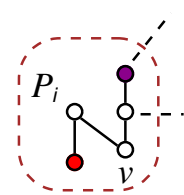

(i)

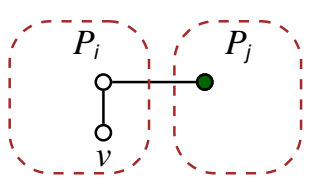

(ii)

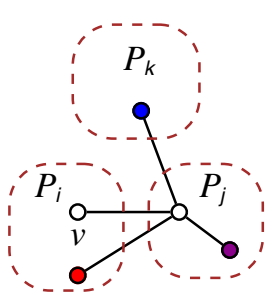

(iii)

Fig. 2. Distribution scenarios of the distance-2 neighbors of vertex $v$ across processors

processors owning their D1 neighbors. To perform the coloring, a processor first gathers information from other processors to build a (partial) list of forbidden colors for each of its boundary vertices scheduled to be colored in the current superstep. Such a list for a vertex $v$ consists of the colors used by its already colored D2 neighbors. The colors of the off-processor vertices in $N(v)$ colored in previous supersteps are easily available since the host processor of $v$ has already received and stored them. We refer to such and on-processor colors as local. However, the colors used by vertices exactly two edges away from $v$ may have to be obtained from another processor.

Figure2 2illustrates the three scenarios in which the vertices on a path $v, w, x$ may be distributed among processors. Case (i) corresponds to the situation where both $w$ and $x$ are owned by $P_{i}$. Case (ii) shows the situation where $w$ is owned by $P_{i}$ and $x$ is owned by $P_{j}, j \neq i$. In these two cases, the color of $w$ is local to $P_{i}$. Case (iii) shows the situation where $w$ is owned by $P_{j}$, and vertices $v$ and $x$ do not have a common D1 neighbor owned by $P_{i}$. Vertex $x$ may be owned by any one of the three processors $P_{i}$, $P_{j}$, or $P_{k}, i \neq j \neq k$. In case (iii), the color of $x$ is not local to $P_{i}$ and needs to be relayed through $P_{j}$ which is capable of detecting the situation. In particular, $P_{j}$ builds and sends a list of forbidden colors for each vertex owned by $P_{i}$ that $P_{i}$ cannot access directly. Since $P_{j}$ does not know the internal structure of the vertices in $P_{i}$, it includes the color of every $x$ in the list of forbidden colors for $v$ for each path $v, w, x$ where $w$ is owned by $P_{j}$.

At the beginning of the algorithm, each processor sends a coloring-schedule of its boundary vertices to neighboring processors. In this way, each processor will know the D2 color information it needs to send in each superstep. Note that it is only necessary to send information regarding D1 neighbors of a vertex owned by another processor. Each processor then computes a list $X_{i}$ of vertices on neighboring processors for which it must supply color information. With the knowledge of $X_{i}$, processor $P_{i}$ can now be "pro-active" in building and sending lists of relevant color information. When a processor receives the partial lists of forbidden colors from all of its neighboring processors, it merges these lists with local color information to determine a complete list of forbidden colors for its vertices scheduled to be colored in the current superstep. Using this information, a processor then speculatively colors these vertices and sends the new color information to processors owning D1 neighbors.

In addition to coloring vertices in the current set $U_{i}$, a processor also computes a list $W_{i}$ of vertices that it needs to examine in the conflict detection phase. Two vertices are involved in a conflict only if they are colored in the same superstep. Thus $W_{i}$ consists 
of (i) every vertex that has at least two neighbors on different processors that are colored in the same superstep, and (ii) every vertex $v$ in $U_{i}$ that has at least one neighbor on a processor $P_{j}, j \neq i$, colored in the same superstep as $v$. The tentative coloring routine sketched so far is outlined with more details in Algorithm 2.

The set $W_{i}$ is efficiently determined in the following manner. The vertices in $X_{i} \cup$ $U_{i}$ are traversed a superstep at a time. For each superstep, first, each vertex in $U_{i}$ and its neighboring boundary vertices are marked. Then for each vertex $v \in X_{i}$ the vertices in $N(v)$ owned by processor $P_{i}$ are marked. If this causes some vertex to be marked twice in the same superstep, then the vertex is added to $W_{i}$. The combined sequential work carried out by $P_{i}$ and its neighboring processors to perform the coloring of $U_{i}$ is $O\left(\Sigma_{v \in U_{i}}|h(v)|\right)$ where $h(v)$ is the graph induced by the edges incident on $N[v]$. Summing over all processors, the total work involved in coloring the vertices in $U=$ $\cup U_{i}$ is $O\left(\Sigma_{v \in U}|h(v)|\right)$ which is equivalent to the complexity of a sequential algorithm.

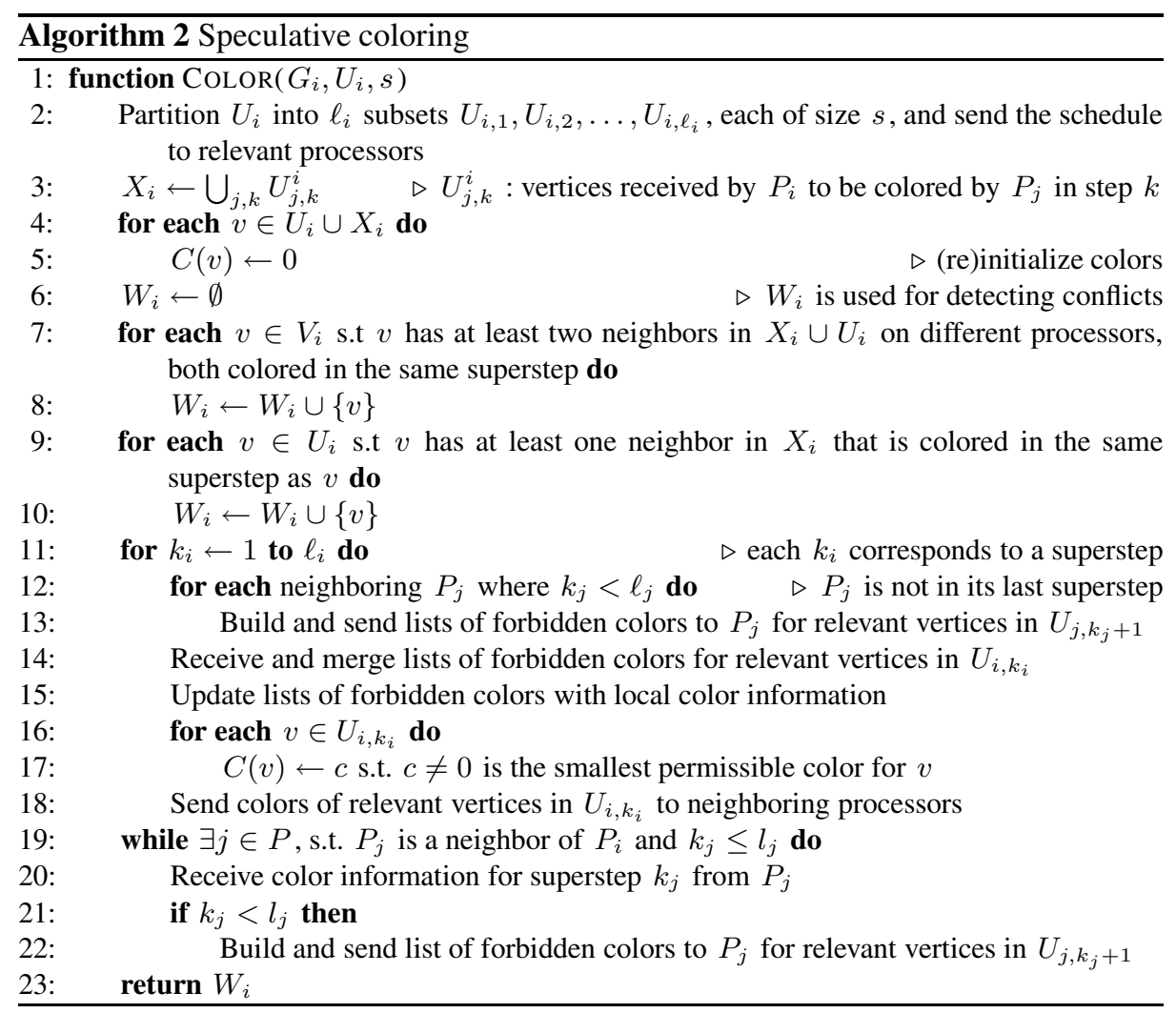

For each $v \in U_{i}$, processor $P_{i}$ receives the colors of vertices exactly two edges from $v$ from processors hosting vertices in $N(v)$. Meanwhile, such processors receive the color of $v$ from $P_{i}$. The only time a color might be sent to $P_{i}$ more than once is when there exists a triangle $v, w, x$ where $w$ is owned by $P_{j}, x$ is owned by $P_{k}$, and 
$i, j$ and $k$ are all different. In such a case, both $P_{j}$ and $P_{k}$ would send the color of $x$ to $P_{i}$. In any case, the overall size of communicated data is bounded by $\Sigma_{v \in \cup U_{i}}|h(v)|$.

The discussion above implies that a partitioning of $\mathcal{G}$ among processors where the number of boundary vertices is small relative to the number of interior vertices on each processor is highly desirable as it reduces communication cost.

\subsection{The Conflict Detection Phase}

A conflict involving a pair of adjacent vertices is detected by both processors owning these vertices. A conflict involving a pair of vertices exactly two edges apart is detected by the processor owning the middle vertex. To resolve a conflict, one of the involved vertices is randomly chosen to be recolored in the next round. Algorithm 3 outlines the parallel conflict detection phase DETECTCONFLICTS executed on each processor $P_{i}$. This routine returns a set of vertices to be colored in the next round by $P_{i}$.

Each processor $P_{i}$ accumulates and sends a list $R_{i, j}$ of vertices to be recolored by each $P_{j}$ in the next round. $P_{i}$ is responsible for recoloring vertices in $R_{i, i}$ and therefore adds received notifications $R_{j, i}$ from each neighboring processor $P_{j}$ to $R_{i, i}$.

To efficiently determine the subset of $W_{i}$ that needs to be recolored, we use two color-indexed tables seen [] and where[]. The assignment seen[c] $=\mathrm{w}$ for a vertex $w \in W_{i}$ is effected if at least one vertex in $\mathrm{N}[\mathrm{w}]$ of color $\mathrm{c}$ has already been encountered. The entry where $[c]$ stores the vertex with the lowest random value among these. Initially both $\operatorname{seen}[C(w)]$ and where $[C(w)]$ are set to $w$. This ensures that any conflict involving $w$ and a vertex in $N(w)$ will be discovered. For each neighbor of $w$, a

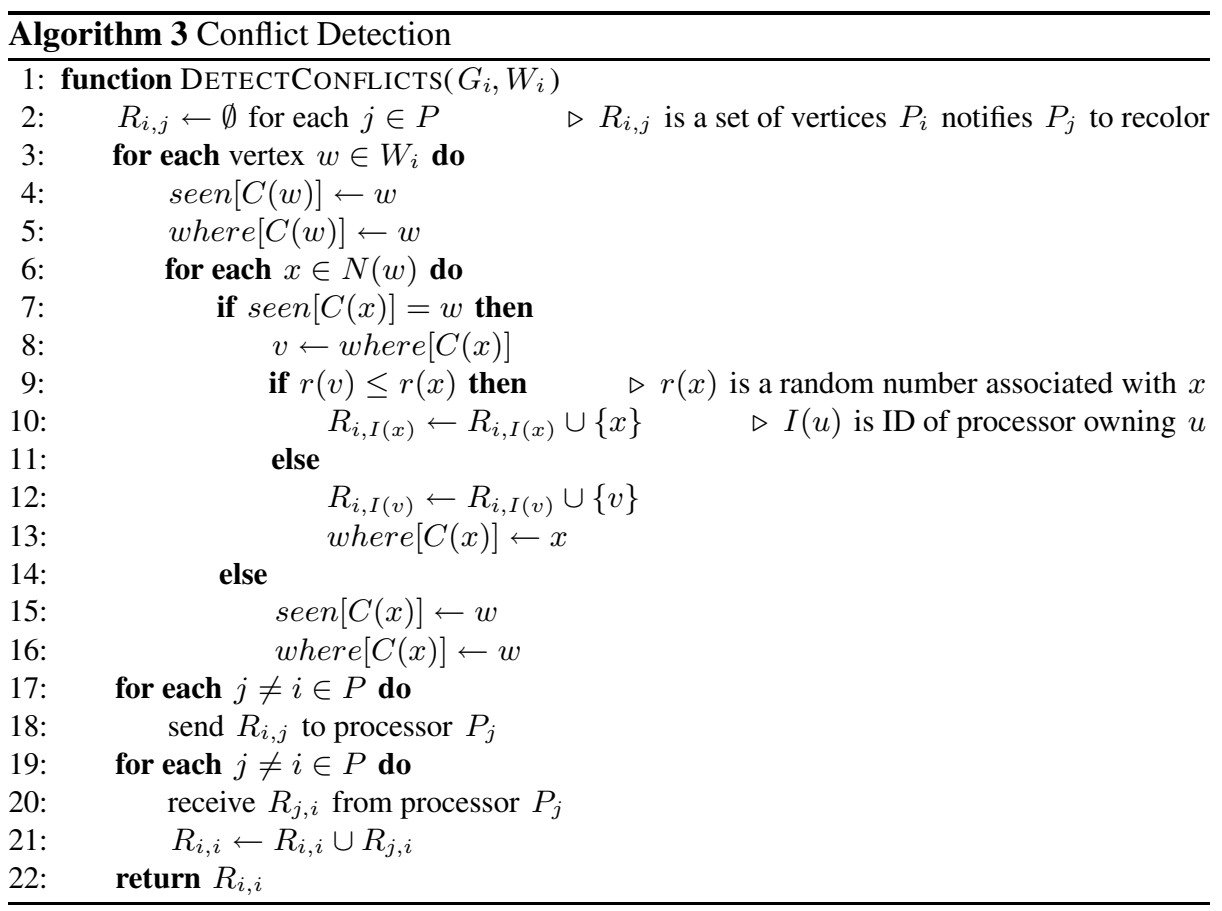


check on whether its color has already been seen is done. If the check turns positive, the vertex that needs to be recolored is determined based on a comparison of random values and the table where is updated accordingly (Lines 3-16).

Note that in Line 6, it is sufficient to only check for conflicts using vertices that are both in $N(w)$ and in either $U_{i}$ or $X_{i}$. However, determining which vertices in $N(w)$ this applies to takes more time than testing for a conflict. Also, it is not necessary to notify a neighboring processor on the detection of a conflict involving adjacent vertices as the conflict will also be discovered by the other processor.

\section{Experimental Results}

We carried out experiments on a 32-node PC cluster equipped with dual $2.4 \mathrm{GHz}$ Intel P4 Xeon CPUs with 4 GB of memory. The nodes are interconnected via a switched $10 \mathrm{Gbps}$ Infiniband network. Our test set consists of 21 graphs from molecular dynamics and finite element applications [11/7|12 13]. We report average results for each class of graphs, instead of individual graphs. Each result is in turn an average of 5 runs.

The left half of Table 4 displays the structural properties of the test graphs. The first part of the right half lists the number of colors and the runtime in milliseconds used by a

Table 1. Structural properties of the test graphs classified according to application area (left). Performance results (right). Sources: MD [13]; FE [7]; CA, SH [11]; ST, AU, CE [12].

\begin{tabular}{|c|c|c|c|c|c|c|c|c|c|c|c|}
\hline \multirow[b]{2}{*}{ app } & \multirow[b]{2}{*}{ ame } & \multirow[b]{2}{*}{$V \mid$} & \multirow[b]{2}{*}{$|E|$} & \multirow[b]{2}{*}{$\begin{array}{l}\text { Degree } \\
\text { max avg }\end{array}$} & \multicolumn{2}{|c|}{$\overline{\mathrm{D} 1}$} & \multicolumn{2}{|c|}{$\overline{\mathrm{D} 2}$} & \multicolumn{3}{|c|}{$\overline{\text { D1 on } \mathcal{G}^{2} \text { (norm.) }}$} \\
\hline & & & & & time & $\mathrm{c}$ & $\begin{array}{r}\text { time } \\
\text { (norn }\end{array}$ & $\mathrm{s}$ & $|\times| E \mid$ & & color. \\
\hline \multirow{3}{*}{$\mathrm{M}$} & & & 255 & 4320 & 3.3 & 21 & 20.6 & 75 & 4.7 & 33.0 & $\overline{4.8}$ \\
\hline & & & & 42 & 5.5 & 19 & 3.2 & 66 & 5.0 & 34.6 & 5.1 \\
\hline & & 92,2 & 6 & 4325 & 16.5 & 20 & 18.1 & 73 & 5.0 & 28.5 & 4.3 \\
\hline \multirow{3}{*}{ FE } & 5 & $\sqrt{46}$ & 1071 & 2615 & 4.3 & $\overline{12}$ & 20.5 & 41 & 4.8 & 25.8 & 4.0 \\
\hline & $3 \mathrm{a}$ & & & 2613 & 5.0 & 11 & 20.9 & 38 & 4.7 & 28.3 & 4.0 \\
\hline & auto & 8,695 & 3,3 & $37 \quad 15$ & 248.9 & 13 & 16.1 & 42 & 4.9 & 16.6 & 4.5 \\
\hline \multirow{3}{*}{ CA } & hor & 2 & & 33549 & 53.3 & 76 & 2.7 & 336 & $\overline{3.2}$ & 5.7 &. \\
\hline & st1 & ,347 & & 43451 & 34.4 & 54 & 3.8 & 435 & 3.3 & 35.6 & 3.0 \\
\hline & ir & 0 & & 4272 & 79.5 & 51 & 70.5 & 843 & 7.0 & 63.8 & 6.2 \\
\hline \multirow{3}{*}{ ST } & $\mathrm{p}$ & $\overline{8}$ & 3 & 7952 & 50.7 & $\overline{48}$ & 5.5 & 180 & 2.9 & 34.8 & 2.7 \\
\hline & sas & & & $275 \quad 48$ & 11.7 & 41 & 2.8 & 276 & 3.2 & 35.5 & 3.2 \\
\hline & ct2 & & & 20651 & 12.5 & 49 & 46.0 & 210 & 3.8 & 37.7 & 3.5 .5 \\
\hline \multirow{3}{*}{$\mathrm{AU}$} & $t_{b}$ & 2 & 5,2 & 7648 & 58.5 & 42 & 5.8 & 103 & 3.2 & 29.2 & 2.7 \\
\hline & oor & ,203 & 22 , & 7648 & 249.7 & 42 & 5.8 & 112 & 3.2 & 29.0 & 2.7 \\
\hline & msdo & & & 7648 & 106.2 & 42 & 36.9 & 105 & 3.2 & 29.8 & 2.7 \\
\hline \multirow{3}{*}{$\mathrm{CE}$} & nloctl & 6 & 2,114 & 8952 & 20.1 & 42 & 43.6 & 126 & 2.9 & 33.0 & 2.7 \\
\hline & & & & 13158 & 23.7 & 66 & 57.6 & 198 & 4.2 & 45.6 & 3.8 \\
\hline & & & & 29969 & 29.2 & 57 & 72.5 & 303 & 6.0 & 62.2 & 6.0 \\
\hline \multirow{3}{*}{ SH } & & & & 10154 & 34.9 & $\overline{48}$ & 48.5 & 126 & 3.1 & 37.7 & 8.2 \\
\hline & & & & 12555 & 46.4 & 50 & 48.5 & 140 & 3.2 & 37.2 & 3.0 \\
\hline & ipsec8 & 14,919 & $3,269,240$ & 13157 & 29.1 & 54 & 52.9 & 150 & 3.5 & 41.2 & 0.4 \\
\hline
\end{tabular}




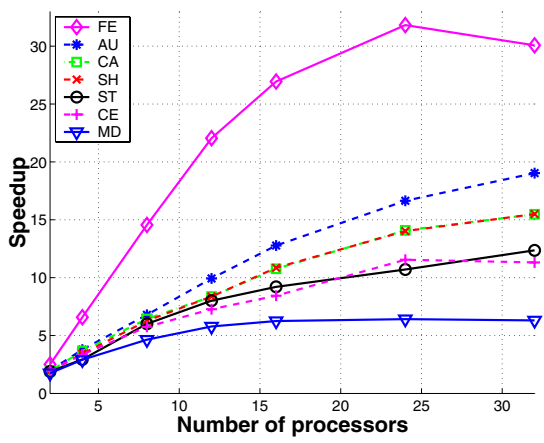

(a)

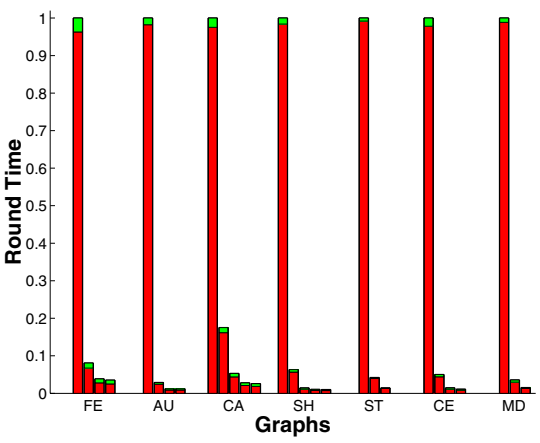

(b)

Fig. 3. (a) Speedup while varying number of processors for $s=100$.(b) Breakdown of execution time into rounds and coloring and conflict detection phases for $p=16$. Each bar is divided into time spent on coloring (bottom) and time spent on conflict detection (top).

sequential D1 coloring algorithm. The second part shows timings for two different ways of sequentially achieving a D2 coloring: a direct D2 coloring on $G$ and a D1 coloring on $G^{2}$. The time spent on constructing $\mathcal{G}^{2}$ from $\mathcal{G}$ is given under column conv. time. The reported times have been normalized with respect to the corresponding time required for performing a D1 coloring. We also list the ratio of the number of edges in $\mathcal{G}^{2}$ to that in $\mathcal{G}$, to show the relative increase in storage requirement.

As one can see, $\mathcal{G}^{2}$ requires a factor of nearly 3 to 7 more storage than $\mathcal{G}$. D2 coloring on $\mathcal{G}$ is in most cases slightly slower than constructing and then D1 coloring $\mathcal{G}^{2}$. We believe this is due to the fact that a D1 coloring on $\mathcal{G}^{2}$ accesses memory more sequentially in comparison with a D2 coloring on $\mathcal{G}$.

Figure 3(a) shows the speedup obtained in using our D2 coloring algorithm on $G$ while keeping the superstep size fixed at 100 . For most graph classes, reasonable speedup is obtained as the number of processors is increased. We have also conducted experiments to investigate the impact of superstep size. We found that with the exception of extreme values, superstep size does not significantly influence speedup.

We observed that the number of conflicts increases with increasing number of processors and superstep size. Still, it stays fairly low and does not exceed $10 \%$ of the number of vertices with the exception of MD graphs with up to 32 processors for $s=100$. The number of rounds the algorithm had to iterate was observed to be consistently low, increasing only slowly with superstep size and number of processors. This is due to the fact that the number of initial conflicts drops rapidly between successive rounds. To further show how the time within each round is spent we present Figure 3(b) The figure shows the time spent on coloring boundary vertices and conflict detection in each round for 16 processors. All timings are normalized with respect to the time spent in the first round, excluding the time spent on coloring interior vertices.

Figure 4(a) shows how the total time is divided into time spent on coloring interior vertices, coloring boundary vertices, and conflict detection. All timings are normalized with respect to the sequential coloring time. As the number of processors increases, the time spent on coloring boundary vertices does not change much while the time spent on coloring interior vertices decreases almost linearly. This should be seen in light of 


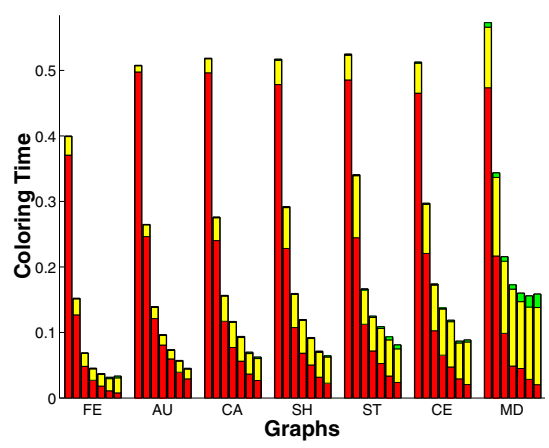

(a) Graphs in Table 4

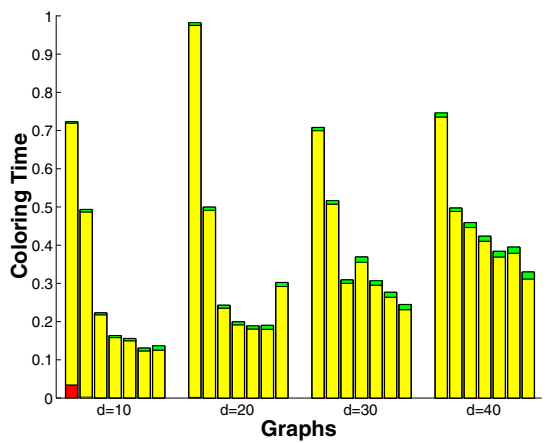

(b) Random graphs

Fig. 4. Breakdown of execution time into time spent on coloring internal vertices (bottom), coloring boundary vertices (middle), and conflict detection (top). For each graph class, timings for $p=2,4,8,12,16,24,32$ are reported.

the fact that the number of boundary vertices increases as more processors are applied whereas the coloring of interior vertices does not involve any communication.

To investigate scalability on boundary vertices, we performed experiments on random graphs. For a random graph almost every vertex becomes a boundary vertex regardless of how the graph is partitioned. We generated random graphs with 100,000 vertices and with average degrees of 10, 20,30, and 40. Figure 4(b) shows that the algorithm scales fairly well and almost all the time is spent on coloring boundary vertices.

We have also evaluated D1 coloring on $\mathcal{G}^{2}$ and experimental results (omitted due to space constraints) indicate that this approach is less scalable and requires more storage than the D2 coloring on $\mathcal{G}$ approach due to large density of $\mathcal{G}^{2}$. We intend to implement parallel construction of $\mathcal{G}^{2}$ and investigate trade-offs between these two approaches in more detail in a future work.

\section{Conclusion}

We have presented an efficient parallel distance- 2 coloring algorithm suitable for distributed memory computers and experimentally demonstrated its scalability. In a future work we plan to adapt the presented algorithm to solve the closely related strong hypergraph coloring problem. This brings up the open problem of finding a suitable partition of the vertices and edges of a hypergraph.

\section{References}

1. Gebremedhin, A.H., Manne, F., Pothen, A.: What color is your jacobian? Graph coloring for computing derivatives. SIAM Rev. (2005) To appear.

2. Krumke, S., Marathe, M., Ravi, S.: Models and approximation algorithms for channel assignment in radio networks. Wireless Networks 7 (2001) $575-584$

3. Vazirani, V.V.: Approximation Algorithms. Springer (2001) 
4. Ferreira, A., Pérennes, S., Richa, A.W., Rivano, H., Stier, N.: Models, complexity and algorithms for the design of multi-fiber wdm networks. Telecommunication Systems 24 (2003) $123-138$

5. McCormick, S.T.: Optimal approximation of sparse hessians and its equivalence to a graph coloring problem. Math. Programming 26 (1983) 153 - 171

6. Boman, E.G., Bozdă̆, D., Catalyurek, U., Gebremedhin, A.H., Manne, F.: A scalable parallel graph coloring algorithm for distributed memory computers. (EuroPar 2005, to appear)

7. Gebremedhin, A.H., Manne, F.: Scalable parallel graph coloring algorithms. Concurrency: Practice and Experience 12 (2000) 1131-1146

8. Gebremedhin, A.H., Manne, F., Woods, T.: Speeding up parallel graph coloring. In: proceedings of Para 2004, Lecture Notes in Computer Science, Springer (2004)

9. Jones, M.T., Plassmann, P.: A parallel graph coloring heuristic. SIAM J. Sci. Comput. 14 (1993) 654-669

10. Gebremedhin, A.H., Manne, F., Pothen, A.: Parallel distance- $k$ coloring algorithms for numerical optimization. In: proceedings of Euro-Par 2002. Volume 2400., Lecture Notes in Computer Science, Springer (2002) 912-921

11. : (Test data from the parasol project) http://www.parallab.uib.no/projects/parasol/data/.

12. : (University of florida matrix collection) http://www.cise.ufl.edu/research/sparse/matrices/.

13. Strout, M.M., Hovland, P.D.: Metrics and models for reordering transformations. In: proceedings of MSP 2004. (2004) 23-34 